\title{
Invasive Lobular Breast Carcinoma with Predominant In Situ Component
}

National Cancer Institute

\section{Source}

National Cancer Institute. Invasive Lobular Breast Carcinoma with Predominant In Situ

Component. NCI Thesaurus. Code C9136.

A breast carcinoma characterized by the presence of a predominant lobular carcinoma in situ component and a minor component of invasive carcinoma. 